\title{
Lipidomic Profiling Reveals Distinct Differences in Plasma Lipid Composition in Overweight or Obesity Adolescence Student
}

Longyan Yang ( $\square$ lyyang15@ccmu.edu.cn )

Beijing Luhe Hospital, Capital Medical University https://orcid.org/0000-0002-0815-5627

Ruili Yin

Beijing Luhe Hospital, Capital Medical University

Xiaojing Wang

Beijing Luhe Hospital, Capital Medical University

$\mathrm{Ke} \mathrm{Yu}$

Beijing Luhe Hospital, Capital Medical University

\section{Research}

Keywords: Lipidomic, Obesity, Overweight, Adolescence

Posted Date: June 25th, 2020

DOI: https://doi.org/10.21203/rs.3.rs-33013/v1

License: (a) (i) This work is licensed under a Creative Commons Attribution 4.0 International License.

Read Full License 


\section{Abstract}

Introduction $\nabla$ The relationship between dyslipidemia and obesity has been widely reported, but the global lipid profiles associated with the development of obesity remain to be clarified, especially in the East Asia teenage population.

Methods $₫$ Mass spectrometry coupled with liquid chromatography was applied to detect the global lipidome in the fasting plasma from 90 Chinese adolescence, including 30 obesity adolescents, 30 overweight adolescents, and 30 adolescents with normal body mass index (BMI). All participants were performed anthropometric measurement by using InBody 770. Clinical biochemical indicators were measured by Cobas Elecsys 601 .

ResultsखBoth qualitative and quantitative analyses revealed a gradual change in plasma lipid features with obesity students exhibiting characteristics close to overweight students, but they differed significantly from students with normal. The levels of triglyceride (TG), 18-Hydroxycortisol, Isohumulinone $\mathrm{A}$, and 11-Dihydro-12-norneoquassin were up-regulated in obesity group, while phosphatidylcholine (PC), phosphatidylethanolamine (PE), LysoPC, LysoPE, and Phosphatidylinositol (PI) were significantly down-regulated in obesity group than in control and overweight individuals. Then conducted venn diagram and selected 8 significant metabolites from the 3 paired comparisons. Most of the selected features significantly correlated with anthropometric measurement.

Conclusions: The altered plasma lipidome in Chinese obesity and overweight students suggests that lipid features may play important role in the pathogenesis of obesity and that such features may provide a basis for evaluating risk and monitoring obesity development.

\section{Background}

The prevalence of obesity and metabolic syndrome can now be observed in both adults and young people. These phenomena affect 380 million children and adolescents worldwide [1]. Childhood obesity have a significant impact on both physical and psychological health [2]. Sahoo et al reported that childhood obesity not only can affect children's physical health, social and emotional well-being, but also self-esteem. Moreover, childhood obesity could lead to metabolic, pulmonary, orthopedic, neurological, cardiovascular, hepatic, and menstrual disorders [3]. WHO defines adolescence as a period of growth and development between the ages of 10 and 19 years after childhood and before adulthood.

It is believed that adolescent and childhood obesity have reached epidemic levels [1], and about $17 \%$ children are facing obesity problem in the United States [2]. The increased prevalence of overweight and obesity in children and adolescents observed in several countries, as weight gain is an independent predictor for metabolic syndrome development although not seen in all obese individuals. Metabolic syndrome is defined as the presence of a combination of risk factors for cardiovascular disease and type 2 diabetes, including obesity, dyslipidemia, hypertension and glucose intolerance [4]. This condition, although more frequent in adults, can manifest at early ages $[5,6]$. Therefore, early diagnosis of possible 
obesity and timely control interventions have a beneficial effect on the health of adults and the prevention of cardiovascular diseases.

By comparing the metabolomics characteristics of obesity, Newgard et al. further revealed resistancerelated BCAA-related metabolite characteristics, and the accompanying specific increase in C3 and C5 carnitine levels indicates an increase in BCAA catabolism [7]. Longitudinal lipidomics studies in children have shown that maternal obesity increases the risk of offspring obesity, which is a long-term change in plasma ceramide levels [8]. To study the changes of metabolites in blood lipids by lipidomics, caloric restriction and improvement of metabolic syndrome following fish oil intake were predicted, and potential lipid metabolites were identified [9]. Pawelzik et al. performed lipidomic analysis of urine samples from obese people and identified the relationship between urinary prostaglandin levels and obesity-related dyslipidemia, abdominal obesity, and insulin resistance [10].

Conventional data-dependent acquisition (DDA) mass spectrometry (MS) mode has been widely used in lipidomic studies, where parameters are detected to minimize duplicate precursor ions and can be optimized to identify complex lipid molecules [11]. However, DDA performance has some inherent limitations, such as limited dynamic range, bias against highly abundant ions, and long duty cycles with increasing sample complexity. A data independent acquisition (DIA) strategy was recently developed to alleviate the limitations of the DDA model [12], it improves detection sensitivity and analytical reproducibility. However, the independent data acquisition method is not easy to apply by lipidomics, because the annotation of MS features and the estimation of false discovery rate in large and complex lipid data sets require more sophisticated software and integrated reference databases [13].

Here, we conducted nontargeted lipidomics analysis of 90 Chinese adolescence students, including 30 obesity students, 30 overweight students, and 30 students with normal BMI, using DIA-based liquid chromatography-tandem mass spectrometry (LC-MS/MS). By using statistical business and in-house software to analyze highly complex data sets, we demonstrate that compared with overweight and normal students, obese students in China have significant changes in lipids in plasma. In addition, we identified several lipid characteristics, including TG, 18-Hydroxycortisol, Isohumulinone A, and 11-Dihydro12-norneoquassin, $\mathrm{PC}, \mathrm{PE}$, LysoPC, LysoPE, and $\mathrm{PI}$, which are potential indicators for predicting obesity risk.

\section{Material And Methods}

\section{Study population}

Nighty teenagers from junior middle school took part in the study (Beijing 9th Middle School, aged between 12 and 13). All the participants signed informed consent with all the measurement in this research which they have to complete. The volunteers with serious diseases and special diet or weight change ( $>2.5 \mathrm{~kg}$ in one month) were excluded. The trial was approved by the Ethic Committee at the Lu- 
he Hospital affiliated Capital Medical University. All participants were included with no change of their eating habits and their habits related to the physical activity.

\section{Data collection and anthropometric measurement}

The participants had to take anthropometric measurement by using InBody 770 (InBody Co. Ltd., Seoul, Republic of Korea). We evaluated the collected data from the anthropometric measurements statistically and graphically in Microsoft Office Excel 2010 (Los Angeles, CA, USA). In this study, blood was allowed to coagulate at $4^{\circ} \mathrm{C}$ and serum was separated by centrifugation for $15 \mathrm{~min}$ at 3,000 rpm. Serum TSH, FT4 and FT3 were tested with an electrochemiluminescence immunoassay (ECLIA) using an Abbott Architect I2000 (Abbott Diagnostics, Abbott Park, IL, USA). Clinical biochemical indicators were measured by Cobas Elecsys 601 (Roche Diagnostics, Switzerland). According to individual BMI values, they were divided into 3 group: values between $16-20 \mathrm{~kg} / \mathrm{m}^{2}$ are control group, values between $20-26 \mathrm{~kg} / \mathrm{m}^{2}$ are overweight group, values between $26-37 \mathrm{~kg} / \mathrm{m}^{2}$ are obesity group. To delineate global lipidomic profiles in Chinese overweight and obesity adolescence, $\mathrm{BMI}$ and body fat percent together with the corresponding clinical and phenotypic data were collected from 3 groups in Beijing, China (Additional file 1).

\section{Liquid chromatography-tandem mass spectrometry (LC- MS/MS)}

Lipids were extracted from individual plasma samples and then injected into the mass instrument in both positive and negative modes, with pooled extraction quality control (QC) samples at certain intervals. In this project, advanced mass spectrometer Xevo G2-XS QTOF (Waters, UK) is used for mass spectrometry data collection, and commercial software PROGENESIS QI (Version 2.2) (Waters, UK) and independently developed metabonomics $\mathrm{R}$ software package metaX are used for statistical analysis of mass spectrometry data, wherein metabolite identification is based on databases HMDB and LipidMaps [14]. Univariate and multivariate analyses were conducted using R statistics software to identify and evaluate the significant metabolites among the groups.

\section{Metabolites extraction method}

$40 \mu \mathrm{L}$ of each sample was added to the corresponding 96-well plate; $120 \mu \mathrm{L}$ of pre-cooled isopropyl alcohol was added, shaken and mixed for $1 \mathrm{~min}$, and then placed in a refrigerator at $-20^{\circ} \mathrm{C}$ for $2 \mathrm{~h}$ or overnight; centrifuged $4000 \mathrm{~g}$ at $4{ }^{\circ} \mathrm{C}$ for $30 \mathrm{~min}$; Placed it in a new 96-well plate and diluted it with $225 \mu \mathrm{L}$ of lipid complex solution (isopropanol: acetonitrile: water $=2: 1: 1$ ); taked $20 \mu \mathrm{L}$ of each sample and mixed it into QC sample; taked $60 \mu \mathrm{L}$ of supernatant Transfer to a 96-well microtiter plate, sealed the label, and tested on the machine.

\section{LC-MS parameters}

All samples were acquired by the LC-MS system followed machine orders. Firstly, all chromatographic separations were performed using an ultra-performance liquid chromatography (UPLC) system (Waters, UK). An ACQUITY UPLC CSH C18 column (100 mm*2.1 mm,1.7 $\mu \mathrm{m}$, Waters凹UK) was used for the separation. The column oven was maintained at $55{ }^{\circ} \mathrm{C}$. The flow rate was $0.4 \mathrm{~mL} / \mathrm{min}$ and the mobile 
phase consisted of solvent A (ACN: H2O = 60:40, 0.1\% formate acid and $10 \mathrm{mM}$ ammonium formate) and solvent $B$ (IPA: $A C N=90: 10,0.1 \%$ formate acid and $10 \mathrm{mM}$ ammonium formate). Gradient elution conditions were set as follows: $0 \sim 2 \min \varangle 40-43 \%$ phase B; $2.1 \sim 7 \min \varangle 50-54 \%$ phase B; $7.1-13$ min, 70-99\% phase B; 13.1-15 min, 40\% phase B. The injection volume for each sample was $10 \mu \mathrm{L}$.

\section{Mass spectrometer description}

A high-resolution tandem mass spectrometer Xevo G2 XS QTOF (Waters, UK) was used to detect metabolites eluted form the column. The Q-TOF was operated in both positive and negative ion modes. For positive ion mode, the capillary and sampling cone voltages were set at $3.0 \mathrm{kV}$ and $40.0 \mathrm{~V}$, respectively. For negative ion mode, the capillary and sampling cone voltages were set at $2 \mathrm{kV}$ and $40 \mathrm{~V}$, respectively. The mass spectrometry data were acquired in Centroid MSE mode. The TOF mass range was from 100 to $2000 \mathrm{Da}$ in positive mode and 50 to $2000 \mathrm{Da}$ in negative mode. And the survey scan time was $0.2 \mathrm{~s}$. For the MS/MS detection, all precursors were fragmented using 19-45 eV, and the scan time was $0.2 \mathrm{~s}$. During the acquisition, the LE signal was acquired every $3 \mathrm{~s}$ to calibrate the mass accuracy. Furthermore, in order to evaluate the stability of the LC-MS during the whole acquisition, appropriate standards were run and a quality control sample (Pool of all samples) was also acquired after every 10 samples.

\section{Results}

\section{Assessment of clinical characteristics and plasma lipidomic features}

The clinical information including physiological and anthropometric indicators of the individuals included in this cohort is summarized in Table 1. The participants were divided into three groups according to their BMI values. The level of SBP, Waist-hip ratio, fat mass, body fat percent and visceral fat area were significantly higher in both overweight and obesity individuals than in control group, with obesity participants exhibiting higher values compared with overweight individuals (Kruskal-Wallis test, $\mathrm{P}<$ 0.001).

We evaluated both coverage and reproducibility of the non-targeted lipidomic data on our sample. Using Progenesis QI 2.0 and metaX, the non-targeted metabolomics analysis yielded 51135 positive ion mode (Additional file 2) and 8988 negative ion mode (Additional file 3).

\section{Overweight and obesity-related features}

Because of the observed effects of obesity on lipid profiles, we performed a blocked Kruskal-Wallis test, using obesity group as the blocking factor, followed by Dunn's hoc test for paired comparisons. As shown in Additional file 4 and 5, 876 in positive and 544 in negative features displayed gradually up-regulated among the 3 groups. Also, there are 1081 in positive and 353 negative features showed down-regulated in Additional file 6 and 7. Of these, there are lipid or lipid-like compounds, also including organooxygen 
compounds, amino acids, peptides, and analogues, benzyl alcohols, glycerophospholipids and triacylglycerol. As shown in Fig. 1, paired comparisons revealed that 460 features (290 features in Additional file 8 positive and 170 features in Additional file 11 in negative) exhibited significant differences between control and obesity group, whereas 231 and 244 features (Additional file 9 and 12, Additional file 10 and 13 in both positive and negative, respectively) showed obvious differences between overweight versus control group and obesity group, respectively $(P<0.05)$. Of these significant changed metabolites, we screened out eight (six positive and two negative) metabolites with significant differences in expression among the three groups. The number of variables distinguishing overweight and obesity suggested that changes in a large fraction of the lipid profiles in overweight and obesity were shared, implying that compared with control group, the overweight and obesity group shares similar metabolites.

To quantify the differential features among the 3 groups, all detected features were assessed using criteria: 1 ) variable importance of the projection $(\mathrm{VIP})>1.0$ estimated by partial least squares discriminant analysis (PLS-DA); 2) fold change in mass intensity $\geq 1.2$ or $\leq 0.83$; 3 ) $P<0.05$.

\section{Table 1}

Basic characteristics of three groups in the study. 


\begin{tabular}{|c|c|c|c|c|c|c|c|}
\hline Variables & $\begin{array}{l}\text { Control } \\
(n= \\
30)\end{array}$ & $\begin{array}{l}\text { Overweight } \\
(n=30)\end{array}$ & $\begin{array}{l}\text { Obesity } \\
(n=30)\end{array}$ & $\begin{array}{l}\mathrm{P} \\
\text { value }^{\mathrm{b}}\end{array}$ & $\begin{array}{l}\text { Obesity vs } \\
\text { Overweight } \\
\text { c }\end{array}$ & $\begin{array}{l}\text { Obesity } \\
\text { vs } \\
\text { Control } \\
\text { c }\end{array}$ & $\begin{array}{l}\text { Overweight } \\
\text { vs Control } \\
\text { c }\end{array}$ \\
\hline $\begin{array}{l}\text { Gender } \\
\text { (female \%), } \\
\text { no. (\%) a }\end{array}$ & $\begin{array}{l}18 \\
(60.00)\end{array}$ & $16(53.33)$ & $\begin{array}{l}14 \\
(46.67)\end{array}$ & 0.594 & -- & -- & -- \\
\hline Age, year & $\begin{array}{l}12.50 \\
\pm 0.51\end{array}$ & $\begin{array}{l}12.73 \pm \\
0.45\end{array}$ & $\begin{array}{l}12.77 \\
\pm 0.47\end{array}$ & 0.058 & 0.958 & 0.072 & 0.132 \\
\hline $\mathrm{BMI}, \mathrm{Kg} / \mathrm{m}^{2}$ & $\begin{array}{l}17.49 \\
\pm 1.41\end{array}$ & $\begin{array}{l}23.76 \pm \\
1.00\end{array}$ & $\begin{array}{l}29.89 \\
\pm 3.17\end{array}$ & $\begin{array}{l}<.0001 \\
0 .\end{array}$ & $<0.0001$ & $<.0001$ & $<0.0001$ \\
\hline SBP, mmHg & $\begin{array}{l}111.93 \\
\pm 9.77\end{array}$ & $\begin{array}{l}120.27 \pm \\
7.18\end{array}$ & $\begin{array}{l}123.13 \\
\pm 6.23\end{array}$ & $\stackrel{<}{0.0001}$ & $<0.0001$ & $\hat{0}_{0.0001}$ & 0.339 \\
\hline $\mathrm{DBP}, \mathrm{mmHg}$ & $\begin{array}{l}68.13 \\
\pm 6.77\end{array}$ & $\begin{array}{l}68.73 \pm \\
4.68\end{array}$ & $\begin{array}{l}70.07 \\
\pm 7.10\end{array}$ & 0.477 & 0.69 & 0.46 & 0.927 \\
\hline $\mathrm{TG}, \mathrm{mmol} / \mathrm{L}$ & $\begin{array}{l}0.88 \pm \\
0.36\end{array}$ & $1.08 \pm 0.67$ & $\begin{array}{l}1.12 \pm \\
0.61\end{array}$ & 0.207 & 0.964 & 0.225 & 0.342 \\
\hline $\mathrm{CHO}, \mathrm{mmol} / \mathrm{L}$ & $\begin{array}{l}4.16 \pm \\
0.76\end{array}$ & $4.03 \pm 0.80$ & $\begin{array}{l}4.34 \pm \\
0.77\end{array}$ & 0.297 & 0.268 & 0.631 & 0.796 \\
\hline $\mathrm{HDL}, \mathrm{mmol} / \mathrm{L}$ & $\begin{array}{l}1.39 \pm \\
0.29\end{array}$ & $1.24 \pm 0.22$ & $\begin{array}{l}1.22 \pm \\
0.20\end{array}$ & 0.015 & 0.885 & 0.018 & 0.061 \\
\hline $\mathrm{LDL}, \mathrm{mmol} / \mathrm{L}$ & $\begin{array}{l}2.32 \pm \\
0.53\end{array}$ & $2.35 \pm 0.61$ & $\begin{array}{l}2.71 \pm \\
0.66\end{array}$ & 0.031 & 0.069 & 0.048 & 0.987 \\
\hline $\begin{array}{l}\text { Waist-hip } \\
\text { ratio }\end{array}$ & $\begin{array}{l}0.79 \pm \\
0.03\end{array}$ & $0.85 \pm 0.04$ & $\begin{array}{l}0.91 \pm \\
0.05\end{array}$ & $\begin{array}{l}< \\
0.0001\end{array}$ & $<0.0001$ & $\begin{array}{l}< \\
0.0001\end{array}$ & $<0.0001$ \\
\hline $\mathrm{FBG}, \mathrm{mmol} / \mathrm{L}$ & $\begin{array}{l}5.52 \pm \\
0.37\end{array}$ & $5.56 \pm 0.42$ & $\begin{array}{l}5.60 \pm \\
0.42\end{array}$ & 0.740 & 0.907 & 0.718 & 0.933 \\
\hline Fat mass, Kg & $\begin{array}{l}9.00 \pm \\
3.33\end{array}$ & $\begin{array}{l}19.70 \pm \\
4.05\end{array}$ & $\begin{array}{l}28.82 \\
\pm 6.96\end{array}$ & $\begin{array}{l}<.0001 \\
0.0\end{array}$ & $<0.0001$ & $\begin{array}{l}<.0001 \\
\end{array}$ & $<0.0001$ \\
\hline $\begin{array}{l}\text { Body fat } \\
\text { percent, \% }\end{array}$ & $\begin{array}{l}19.86 \\
\pm 6.06\end{array}$ & $\begin{array}{l}31.63 \pm \\
5.44\end{array}$ & $\begin{array}{l}38.00 \\
\pm 6.60\end{array}$ & $\begin{array}{l}<.0001 \\
0.0\end{array}$ & $<0.0001$ & $\begin{array}{l}< \\
0.0001\end{array}$ & $<0.0001$ \\
\hline $\begin{array}{l}\text { Viseral fat } \\
\text { area, } \mathrm{cm}^{2}\end{array}$ & $\begin{array}{l}38.78 \\
\pm 14.26\end{array}$ & $\begin{array}{l}88.76 \pm \\
24.86\end{array}$ & $\begin{array}{l}138.81 \\
\pm 39.78\end{array}$ & < 0.0001 & $<0.0001$ & $\hat{0}_{0.0001}$ & $<0.0001$ \\
\hline
\end{tabular}

a $P$ value of chi-square test.

b P value of Kruskal-Wallis test. 
c P-value of Dunn's post hoc test.

Comparision between control and overweight, overweight and obesity, and cntrol and obesity using random forest classifier and ROC curve

As the qualitative and quantitative analyses revealed significant differences in the metabolites levels between the 3 groups and indicated a gradual change from control to obesity via overweight, we investigated if the metabolites could predict risk of further obesity development. To assess this possibility, we used a random forest classifier.

As illustrate in Fig. 1, 8 metabolites were generated. The relationships among the 3 groups were analyzed by random forest classifier and receiver operating characteristic (ROC) curve. Figure 2A-C showed that an area under the ROC curve (AUC) is $61.90 \%$ (95\% confidence interval $(\mathrm{Cl})=42.00-85.60 \%), 62.80 \%(95 \%$ $\mathrm{Cl}=21.50-86.50 \%), 74.30 \%(95 \% \mathrm{Cl}=56.00-91.00 \%)$ between control and overweight, overweight and obesity, and control and obesity in down-regulated both positive and negative ion mode. For up-regulated, the AUC is $59.70 \%(95 \% \mathrm{Cl}=19.50-82.50 \%), 65.40 \%(95 \% \mathrm{Cl}=34.10-75.50 \%), 72.10 \%(95 \% \mathrm{Cl}=49.00-$ $93.50 \%)$ in Fig. 2D-F. Together, these results indicate that the lipidomic profiles are regulated in a complex manner during development of overweight and obesity.

(A-F) ROC and AUC for validation set with Control and Overweight, Overweight and Obesity, and Control and Overweight, respectively. The model was trained using decreased and increased intensity of the detected features from positive and negative ion mode

in the training set among control, overweight and obesity $(n=30)$.

\section{The level of selected metabolites in control, overweight and obesity groups}

As illustrated in Fig. 1, 8 metabolites were selected from both positive and negative ion mode lipidomic profiling. Then the expression of selected metabolites was shown in Fig. 3. Figure $3 \mathrm{~A}$ and Fig. 3B indicated that 6.10_861.5490 m/z and 1.82_480.3095 m/ $\mathrm{z}$ in negative ion mode were gradually decreased in control, overweight and obesity groups. Figure 3D and Fig. 3H exhibited 1.11_396.2412 m/z and10.13_949.7263 m/z in selected positive ion mode were gradually increased in control, overweight and obesity groups. However, 4.86_902.5761 m/z was gradually decreased in Fig. 3E, 4.84_530.4012n and 4.96_546.3962n peaked in overweight group (Fig. 3F-G). In summary, the development of obesity may go through the process of overweight in most cases, but it may directly develop into obesity through the alterations of some lipid metabolites.

(A) and (B) showed negative ion modes level in control, overweight and obesity groups. (C-G) showed positive ion modes level in control, overweight and obesity groups.

\section{Correlations between selected metabolites with clinical parameters}


In the body of overweight and obese people, metabolism is inevitably changed. Hence, the metabolites were also changed. To investigate the relationship between selected metabolites and clinical parameters, we performed a correlation analysis between them. As shown in Fig. 4A, 6.10_861.5490 m/z was negatively with BMI, visceral fat area, body fat percent, and waist/hip ratio. 1.82_480.3095 m/z was negatively with $\mathrm{BMI}$, visceral fat area, body fat percent, and waist/hip ratio, but positively with triglyceride in Fig. 4B. 4.84_530.4012n was negatively with total cholesterol (CHO) in Fig. 4C. 1.11_396.2412 m/z was positively with BMI, visceral fat area, and waist/hip ratio in Fig. 4D. 4.86_902.5761 m/z was negatively with BMI, but positively with triglyceride. (Fig. 4E) 10.13_949.7263 m/z was positively with $\mathrm{BMI}$, visceral fat area, waist/hip ratio, triglyceride, and body fat percent.

1. 6.10_861.5490 $\mathrm{m} / \mathrm{z}$ was negatively with $\mathrm{BMI}$, visceral fat area, body fat percent, waist/hip ratio, and HDL. (B) 1.82_480.3095 m/z was negatively with BMI, visceral fat area, body fat percent, and waist/hip ratio. (C) 4.84_530.4012n was negatively with cholesterol. (D) 1.11_396.2412 m/z was positively with BMI, visceral fat area, body fat percent, and waist/hip ratio. (E) 4.86_902.5761 m/z was negatively with BMI, but positively with triglyceride. (F) 10.13_949.7263 m/z was positively with BMI, body fat percent, visceral fat area,

2. FT3, waist/hip ratio, and triglyceride.

Phospholipids phosphatidylcholine (PC) and phosphatidylethanolamine (PE) are the two most abundant phospholipid species in eukaryotic cells [15]. Lysophosphatidylcholine (LysoPC), an important signaling molecule and fatty acid carrier, constitutes $5-20 \%$ of total plasma phospholipids [16]. Phosphatidylinositol (PI) plays an important role in cell morphology, metabolic regulation, signal transduction and various physiological functions. 1.82_480.3095 m/z was annotated as PC (15:0/0:0), PE (18:0/0:0), LysoPC (15:0), and LysoPE (0:0/18:0). 6.10_861.5490 m/z was annotated as PI (14:0/22:2(13Z, 16Z))- PI (22:2(13Z,16Z)/14:0) (Additional file 3). 1.11_396.2412 m/z was annotated as 18-Hydroxycortisol, Isohumulinone A, and 11-Dihydro-12-norneoquassin; 4.86_902.5761 m/z was annoted as PI (18:0/20:5 (5Z,8Z,11Z,14Z,17Z)); 10.13_949.7263 m/Z was annoted as TG (20:4 $(5 Z, 8 Z, 11 Z, 14 Z) / 20: 3(5 Z, 8 Z, 11 Z) / 18: 3(9 Z, 12 Z, 15 Z))$. The levels of TG, 18-Hydroxycortisol, Isohumulinone $A$, and 11-Dihydro-12-norneoquassin were up-regulated in obesity group, while $P C, P E$, LysoPC, LysoPE, and PI were significantly down-regulated in obesity group than in control and overweight individuals (Additional file 2).

\section{Discussion}

Due to the increased prevalence of obesity in children and adolescents, various studies have been conducted to discover which associations and risk factors increase the likelihood of obesity in children. Although it is still difficult to fully grasp all the risk factors related to obesity, it is of great significance to control and prevent obesity by combining diet, exercise, physiological factors and psychological factors [2]. The short-term and long-term effects of obesity on children's health are a major issue due to adverse psychological and health consequences [17]. Potential negative psychological outcomes are depressive symptoms, poor body image, low self-esteem, risk of eating disorders, and behavioral and learning 
problems; negative health consequences include insulin resistance, type 2 diabetes, asthma, hypertension, and nonalcoholic steatohepatitis $[17,18]$. Obese children are more likely to become obese adults, and therefore increase their risk of multiple diseases before they even reach puberty [18].

The human lipidomic profile reflects lipid metabolism, including the early phase of pathophysiological changes associated with diseases. Wang et al. observed a significant reduction in the levels of five lysophosphatidylcholines (LPC) species (LPC18:2, LPC18:1, LPC20:2, LPC20:1, and LPC20:0) in the obese group compared with the normal-weight group [19]. In addition, lower total LPC, LPC18:0, LPC18:2, and LPC20:4 levels were measured in obese and obese subjects with type 2 diabetes than in nonobese adults. A difference in the LPC profile was not observed between obese individuals and obese subjects with type 2 diabetes [20]. Moreover, Wallace et al. reported associations between the levels of several LPC species, BMI, and inflammatory markers [21]. The authors identified higher levels of LPC14:0 and LPC18:0 and a lower concentration of LPC18:1 in obese subjects compared with lean subjects [22].

Obesity can be estimated in several ways: Body mass index (BMI), the ratio of weight to squared height [23], is used as the most common indicator of obesity. It is convenient and simple, but it can cause changes in cardiovascular and metabolic performance between individuals, but there are alternative methods of body fat distribution. Higher WHR indicates more intraperitoneal cavity and is associated with a higher risk of type 2 diabetes, cardiovascular disease and mortality [24]]. At the same time, waist circumference can also be used. Similar to WHR [25], it is considered a more direct and reliable method. Generally, body fat percentage (BFP) is a method in the body to measure the ratio of adipose tissue to lean meat and water [26], and most are determined using bioelectrical impedance. BFP is not related to $\mathrm{BMI}$, it is associated with an increase in all-cause mortality, and it is generally suggested to estimate obesity better than BMI [27]. Therefore, this study aimed at Chinese teenagers, a group with relatively stable diet and lifestyle, carried out a lipidomics study to observe the development process of obesity and to screen out some biochemical indicators for predicting obesity.

In the present study, the levels of TG, 18-Hydroxycortisol, Isohumulinone A, and 11-Dihydro-12norneoquassin were up-regulated in obesity group, while PC, PE, LysoPC, LysoPE, and PI were significantly down-regulated in obesity group than in control and overweight individuals. 1.82_480.3095 m/z was annotated as PC (15:0/0:0), PE (18:0/0:0), LysoPC (15:0), and LysoPE (0:0/18:0). 6.10_861.5490 m/z was annotated as PI (14:0/22:2(13Z, 16Z))- PI (22:2(13Z,16Z)/14:0) (Additional file 3). According to the Fig. 1, eight metabolites generated only in 1.11_396.2412 m/z was annotated as 18-Hydroxycortisol, Isohumulinone A, and 11-Dihydro-12-norneoquassin; 4.86_902.5761 m/Z was annoted as PI (18:0/20:5 (5Z,8Z,11Z,14Z,17Z)); 10.13_949.7263 m/z was annoted as TG $(20: 4(5 Z, 8 Z, 11 Z, 14 Z) / 20: 3(5 Z, 8 Z, 11 Z) / 18: 3(9 Z, 12 Z, 15 Z))$ (Additional file 2). These data suggested that the development of obesity may does not have to be through overweight, and it may develop directly to obesity due to some changes in lipid metabolism.

There are also some limitations in our study. First of all, it was a cross-sectional study which only addressed the alterations of lipidomic profiling in normal, overweight and obesity students. Furthermore, 
subjects were selected into groups just according to BMI rather than selected randomly, therefore, this can produce selection bias. In addition, there is a small sample study. So based on the above limitations, more large-scale population studies are still needed in the future investigation.

\section{Conclusions}

In summary, the altered plasma lipidome in Chinese obesity and overweight students suggests that lipid features may play important role in the pathogenesis of obesity and that such features may provide a basis for evaluating risk and monitoring obesity development.

\section{Abbreviations}

BMI: body mass index; SBP: systolic pressure; DBP: diastolic pressure; TG: triglyceride; $\mathrm{CHO}$ : cholesterol; HDL: high density lipoprotein; LDL: Low density lipoprotein; FBG: fast blood glucose. PC: phosphatidylcholine; PE: phosphatidylethanolamine; PI: phosphatidylinositol; DDA: data-dependent acquisition; DIA: data-independent acquisition; LC-MS/MS: liquid chromatography-tandem mass spectrometry; MS: mass spectrometry; UPLC-MS: ultra-performance liquid chromatography-mass spectrometry; VIP: variable importance of the projection.

\section{Declarations}

\section{Ethical Approval and Consent to participate}

All the participants signed informed consent with all the measurement in this research, and the trial was approved by the Ethic Committee at the Beijing Luhe Hospital affiliated Capital Medical University.

\section{Consent for publication}

All authors agree to publish this article in the journal of Lipids in Health and Disease.

\section{Availability of data and material}

The author has produced the original data described in the manuscript, which can be obtained free of charge by any scientist who wants to use it, without violating the confidentiality rules of the participants.

\section{Competing interests}

The authors declare that they have no conflict of interests.

\section{Funding}

This study was funded by the Beijing Natural Science Foundation (Project Number: 7184222) and National Natural Science Foundation of China (Project Number: 81800768). 


\section{Authors' contributions}

LyY performed data analysis and interpretation and wrote the first draft of the manuscript. RIY conducted the research and contributed to data interpretation and writing and editing the manuscript. DZ is the chief investigator of the trial and planned and designed the study, obtained funding, oversaw data collection and analysis, and contributed to data interpretation and writing and editing the manuscript. KY takes responsibility for data integrity and accuracy. All authors meet the ICMJE criteria for authorship, and have approved the final version of the manuscript.

\section{Acknowledgements}

We thank associate professor Dong Zhao at Beijing Luhe Hospital for providing available condition to collect information of all participants.

\section{Conflicts of interest}

The authors declare that they have no conflict of interests.

\section{References}

1. Salamonowicz MM, Zalewska A, Maciejczyk M. Oral consequences of obesity and metabolic syndrome in children and adolescents. Dent Med Probl. 2019;56(1):97-104.

2. Sanyaolu A, Okorie C, Qi X, Locke J, Rehman S. Childhood and Adolescent Obesity in the United States: A Public Health Concern. Glob Pediatr Health. 2019;6:2333794 × 19891305.

3. Sahoo K, Sahoo B, Choudhury AK, Sofi NY, Kumar R, Bhadoria AS. Childhood obesity: causes and consequences. J Family Med Prim Care. 2015;4(2):187-92.

4. Pietrobelli A, Malavolti M, Battistini NC, Fuiano N. Metabolic syndrome: a child is not a small adult. Int J Pediatr Obes. 2008;3(Suppl 1):67-71.

5. Chissini RBC, Kuschnir MC, de Oliveira CL, Giannini DT, Santos B. Cutoff values for HOMA-IR associated with metabolic syndrome in the Study of Cardiovascular Risk in Adolescents (ERICA Study). Nutrition. 2019;71:110608.

6. de Assuncao Bezerra MK, Freese de Carvalho E, Souza Oliveira J, Pessoa Cesse EA, Cabral de Lira PI, Galvao Tenorio Cavalcante J. Sa Leal V. Health promotion initiatives at school related to overweight, insulin resistance, hypertension and dyslipidemia in adolescents: a cross-sectional study in Recife, Brazil. BMC Public Health. 2018;18(1):223.

7. Newgard CB, An J, Bain JR, Muehlbauer MJ, Stevens RD, Lien LF, Haqq AM, Shah SH, Arlotto M, Slentz CA, et al. A branched-chain amino acid-related metabolic signature that differentiates obese and lean humans and contributes to insulin resistance. Cell Metab. 2009;9(4):311-26.

8. Leon-Aguilar LF, Croyal M, Ferchaud-Roucher V, Huang F, Marchat LA, Barraza-Villarreal A, Romieu I, Ramakrishnan U, Krempf M, Ouguerram K, et al. Maternal obesity leads to long-term altered levels of 
plasma ceramides in the offspring as revealed by a longitudinal lipidomic study in children. Int $J$ Obes (Lond). 2019;43(6):1231-43.

9. Shabrina A, Tung TH, Nguyen NTK, Lee HC, Wu HT, Wang W, Huang SY. n-3 PUFA and caloric restriction diet alters lipidomic profiles in obese men with metabolic syndrome: a preliminary open study. Eur J Nutr. 2019.

10. Pawelzik SC, Avignon A, Idborg H, Boegner C, Stanke-Labesque F, Jakobsson PJ, Sultan A, Back M. Urinary prostaglandin D2 and E2 metabolites associate with abdominal obesity, glucose metabolism, and triglycerides in obese subjects. Prostaglandins Other Lipid Mediat. 2019;145:106361.

11. Kofeler HC, Fauland A, Rechberger GN, Trotzmuller M. Mass spectrometry based lipidomics: an overview of technological platforms. Metabolites. 2012;2(1):19-38.

12. Mapstone M, Cheema AK, Fiandaca MS, Zhong X, Mhyre TR, MacArthur LH, Hall WJ, Fisher SG, Peterson DR, Haley JM, et al. Plasma phospholipids identify antecedent memory impairment in older adults. Nat Med. 2014;20(4):415-8.

13. Hu T, Zhang JL. Mass-spectrometry-based lipidomics. J Sep Sci. 2018;41(1):351-72.

14. Wen B, Mei Z, Zeng C, Liu S. metaX: a flexible and comprehensive software for processing metabolomics data. BMC Bioinformatics. 2017;18(1):183.

15. Gibellini F, Smith TK. The Kennedy pathway-De novo synthesis of phosphatidylethanolamine and phosphatidylcholine. IUBMB Life. 2010;62(6):414-28.

16. Virtanen JA, Cheng KH, Somerharju P. Phospholipid composition of the mammalian red cell membrane can be rationalized by a superlattice model. Proc Natl Acad Sci U S A. 1998;95(9):49649.

17. Skinner AC, Steiner MJ, Henderson FW, Perrin EM. Multiple markers of inflammation and weight status: cross-sectional analyses throughout childhood. Pediatrics. 2010;125(4):e801-9.

18. Lakshman R, Elks CE, Ong KK. Childhood obesity. Circulation. 2012;126(14):1770-9.

19. Wang Y, Jiang CT, Song JY, Song QY, Ma J, Wang HJ. Lipidomic Profile Revealed the Association of Plasma Lysophosphatidylcholines with Adolescent Obesity. Biomed Res Int. 2019; 2019:1382418.

20. Barber MN, Risis S, Yang C, Meikle PJ, Staples M, Febbraio MA, Bruce CR. Plasma lysophosphatidylcholine levels are reduced in obesity and type 2 diabetes. PLoS One. 2012;7(7):e41456.

21. Wallace M, Morris C, O'Grada CM, Ryan M, Dillon ET, Coleman E, Gibney ER, Gibney MJ, Roche HM, Brennan L. Relationship between the lipidome, inflammatory markers and insulin resistance. Mol Biosyst. 2014;10(6):1586-95.

22. Kim JY, Park JY, Kim OY, Ham BM, Kim HJ, Kwon DY, Jang Y, Lee JH. Metabolic profiling of plasma in overweight/obese and lean men using ultra performance liquid chromatography and Q-TOF mass spectrometry (UPLC-Q-TOF MS). J Proteome Res. 2010;9(9):4368-75.

23. Keys A, Fidanza F, Karvonen MJ, Kimura N, Taylor HL. Indices of relative weight and obesity. J Chronic Dis. 1972;25(6):329-43. 
24. Pischon T, Boeing H, Hoffmann K, Bergmann M, Schulze MB, Overvad K, van der Schouw YT, Spencer E, Moons KG, Tjonneland A, et al. General and abdominal adiposity and risk of death in Europe. N Engl J Med. 2008;359(20):2105-20.

25. Visscher TL, Seidell JC, Molarius A, van der Kuip D, Hofman A, Witteman JC. A comparison of body mass index, waist-hip ratio and waist circumference as predictors of all-cause mortality among the elderly: the Rotterdam study. Int J Obes Relat Metab Disord. 2001;25(11):1730-5.

26. Calling S, Hedblad B, Engstrom G, Berglund G, Janzon L. Effects of body fatness and physical activity on cardiovascular risk: risk prediction using the bioelectrical impedance method. Scand $\mathrm{J}$ Public Health. 2006;34(6):568-75.

27. Padwal R, Leslie WD, Lix LM, Majumdar SR. Relationship Among Body Fat Percentage, Body Mass Index, and All-Cause Mortality: A Cohort Study. Ann Intern Med. 2016;164(8):532-41.

\section{Figures}

(A)


(B)
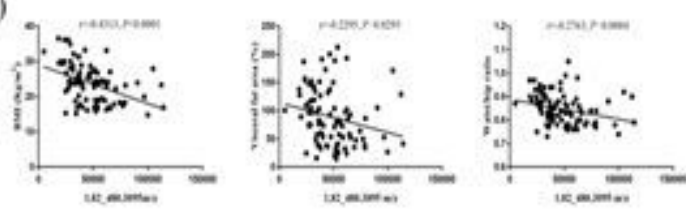

(C)

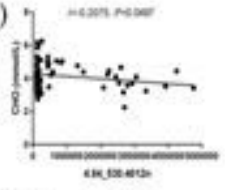

(D)
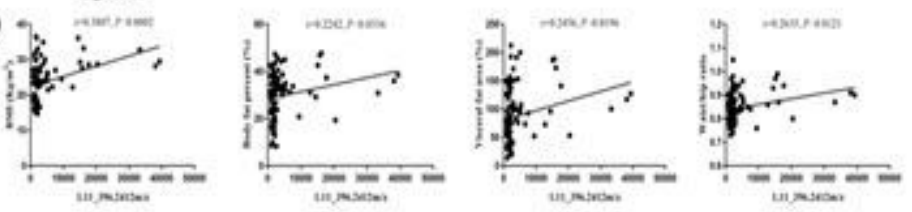

(E)
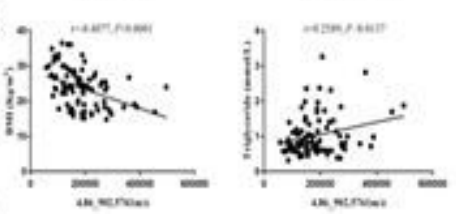

(F)
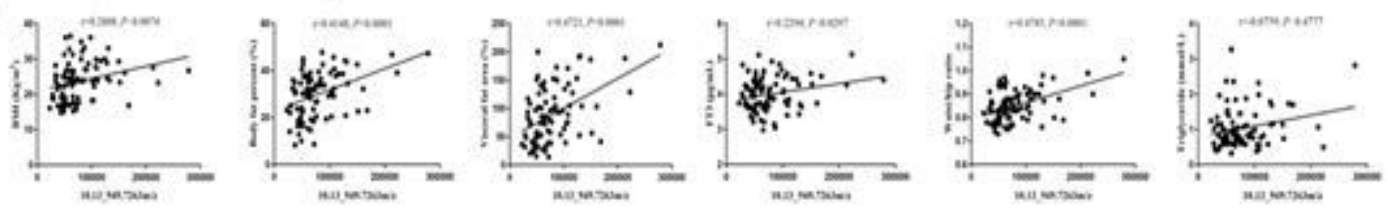

Figure 1 
Correlation between clinical parameters and selected features. (A) $6.10 \_861.5490 \mathrm{~m} / \mathrm{z}$ was negatively with BMI, visceral fat area, body fat percent, waist/hip ratio, and HDL. (B) $1.82 \_480.3095 \mathrm{~m} / \mathrm{z}$ was negatively with BMI, visceral fat area, body fat percent, and waist/hip ratio. (C) 4.84_530.4012n was negatively with cholesterol. (D) 1.11_396.2412m/z was positively with BMI, visceral fat area, body fat percent, and waist/hip ratio. (E) 4.86_902.5761 m/z was negatively with BMI, but positively with triglyceride. (F) 10.13_949.7263m/z was positively with BMI, body fat percent, visceral fat area, (B) FT3, waist/hip ratio, and triglyceride.

(A)

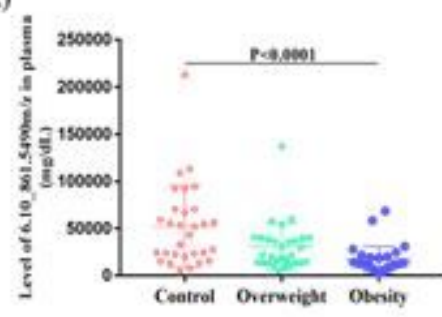

(C)

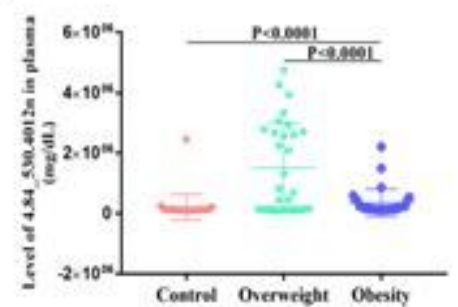

(F)

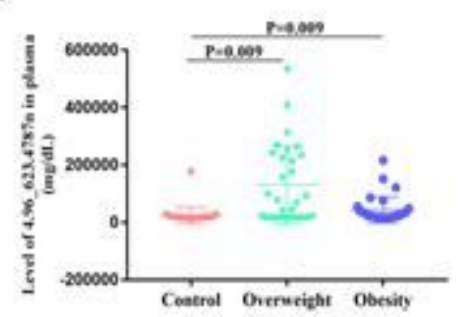

(B)



(D)

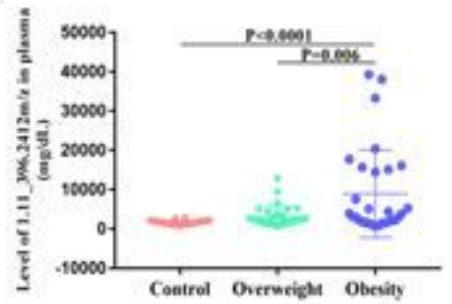

(G)

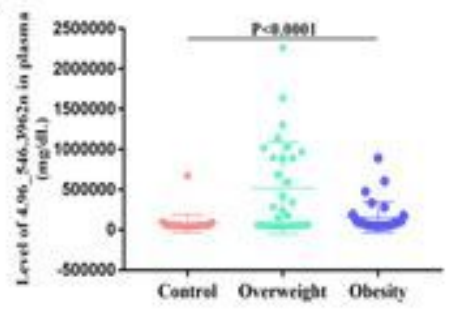

(E)

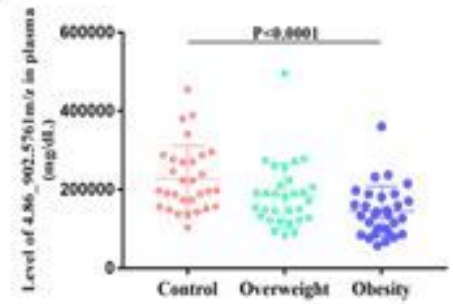

(H)

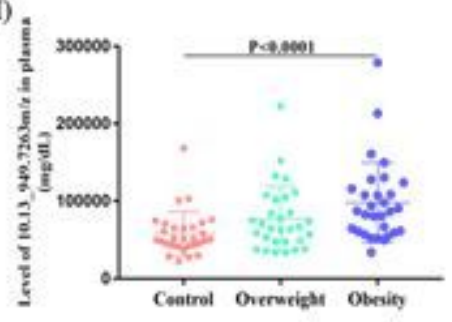

\section{Figure 2}

Level of selected metabolites in control, overweight and obesity groups. (A) and (B) showed negative ion modes level in control, overweight and obesity groups. (C-G) showed positive ion modes level in control, overweight and obesity groups. 
(A)

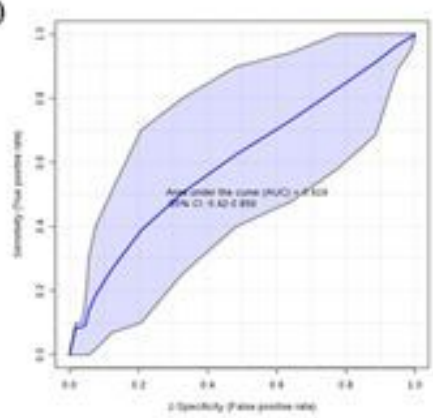

Control vs. Overweight down

(D)

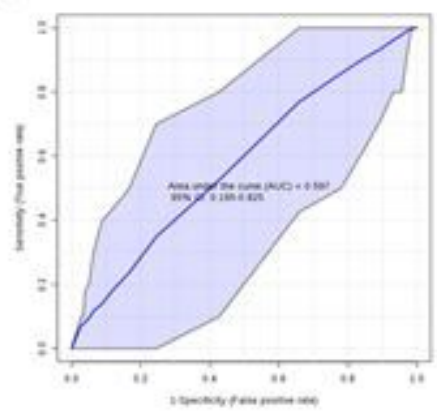

Control vs. Overweight

up
(B)

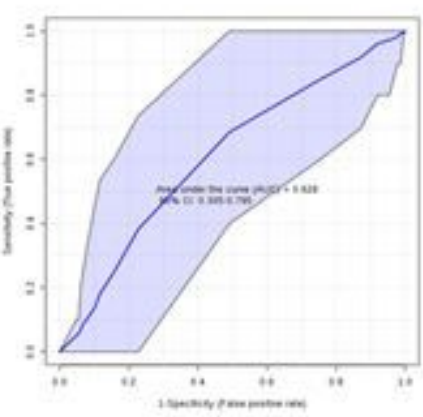

Obesity vs. Overweight down

(E)

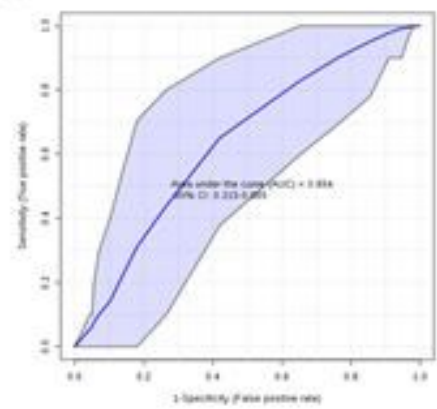

Obesity vs. Overweight up
(C)

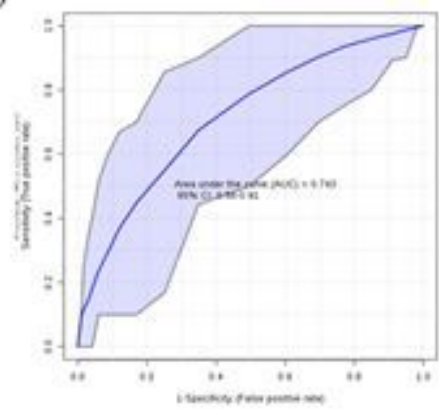

Obesity vs. Control down

(F)



Obesity vs. Control

up

\section{Figure 3}

Receiver operating characteristic curve and area under the ROC curve in the training set. 


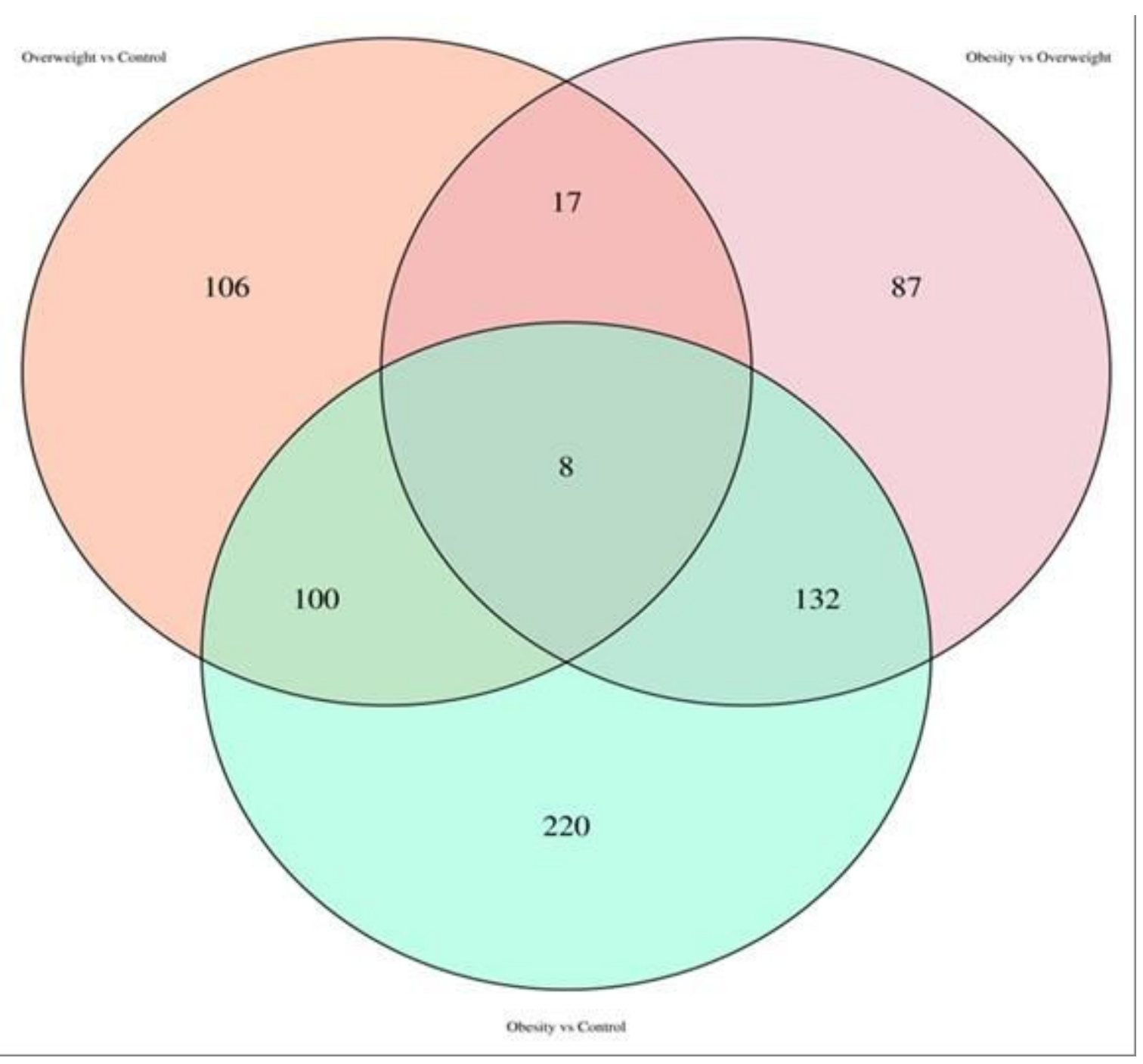

\section{Figure 4}

Venn diagram of significant metabolites from the 3 paired comparisons. Venn diagram depicting the number of significant metabolic features from 3 paired comparisons (the direction of change was ignored, $P<0.05$, Dunn's post hoc test).

\section{Supplementary Files}

This is a list of supplementary files associated with this preprint. Click to download.

- Additionalfile1.xls

- Additionalfile2.xls

- Additionalfile3.xls

- Additionalfile4.xls

- Additionalfile5.xls

- Additionalfile6.xls 
- Additionalfile7.xls

- Additionalfile8.xIs

- Additionalfile9.xls

- Additionalfile10.xls

- Additionalfile11.xls

- Additionalfile12.xls

- Additionalfile13.xls

- coverletter.doc 